\title{
MINMAX PROBLEMS FOR FRACTIONAL PARTS OF REAL NUMBERS
}

\author{
SEON-HONG KIM
}

Abstract. The view-obstruction problem for the $n$-dimensional cube with side 1 can be interpreted as the problem of evaluating the function $\kappa(n)=\inf \max _{0 \leqslant x \leqslant 1} \min _{1 \leqslant k \leqslant n}\left\|v_{k} x\right\|$, where the infimum is taken over all $n$-tuples $v_{1}, \cdots, v_{n}$ of positive integers. So the following could perhaps be called "generalized view-obstruction problems": given a periodic function $\phi$, an interval $I$ and a set of integers $\mathscr{S}$, find

$$
\text { (i) } \min _{x \in I} \max _{s \in \mathscr{S}} \phi(s x), \quad \text { (ii) } \max _{x \in I} \min _{s \in \mathscr{S}} \phi(s x) .
$$

We study minmax problems of this nature where

$$
\phi(x)=\{x\}^{\alpha}(1-\{x\}) \text { and }\left\{(x-1 / 2)^{\alpha}\right\},
$$

and

$$
I=[0,1], \mathscr{S}=\{1, \cdots, N\} .
$$

Here $\{x\}$ denotes the fractional part of $x$, and $N \geqslant 2$ and $\alpha \geqslant 1$ are integers.

Mathematics subject classification (2000): 11J54, 05C35.

Key words and phrases: View-obstruction problem, minmax, fractional parts.

\section{REFERENCES}

[1] T. W. CusicK, View-obstruction problems, Aequationes Math. 9 (1973), 165-170.

[2] T. W. CusicK, View-obstruction problems II, Proc. Amer. Math. Soc. 84 (1982), 25-28.

[3] J. M. WILLS, Zur simultanen homogenen diophantischen Approximation I, Monatsh. Math. 72 (1968), 254-263. 